\title{
Using Bayesian response surface updating for estimating the covariance function of random fields based on limited measurements
}

\author{
P. Criel, R. Caspeele \& L. Taerwe \\ Magnel Laboratory for Concrete Research, Department of Structural Engineering, \\ Faculty of Engineering and Architecture, Ghent University, Belgium
}

\begin{abstract}
Bayesian statistics can be used to update previously obtained information regarding parameters of prediction models. Observed data is often modelled by a nonlinear function of multiple model parameters. Markov Chain Monte Carlo (MCMC) simulations can be used to incorporate these phenomena in the response surface estimation. Formulas as well as an numerical algorithm are presented in order to update the parameters of such response surfaces. Furthermore, the Bayesian response surface updating procedure is applied in order to update the parameters of covariance functions for random fields based on a limited number of available measurements. When there is a lack of sufficient data to effectively apply traditional methods such as the least-squares method (e.g. in case of destructive testing), the parameters of the covariance function are often based on expert judgment. However, a Bayesian updating methodology enables to estimate these parameters more rigorously and with less ambiguity. More specifically, the above mentioned Bayesian response surface updating approach using MCMC simulations is used for this. Prior information can be incorporated in the form of vague or informative priors, which may be based on previous results or expert judgment. The proposed estimation procedure is evaluated trough numerical simulations and compared to the least square (LSQ) method.
\end{abstract}

\section{INTRODUCTION}

It is widely known that material properties have a significant spatial variability due to factors such as effects of workmanship, material heterogeneity and environmental influences. Hence, it is sometimes insufficient to base reliability-based calculations on a random estimate of a homogeneous field of material property values.

Random field theory can be used to model this spatial variability if the covariance function of the field is known. This allows to model material properties (e.g. those of concrete) in a more realistic way,

However, sufficient data to estimate the covariance function is seldom available, especially with respect to properties assessed trough destructive testing. Hence, assumptions with respect to the spatial variability are often made based on expert judgment without consideration of the measurement data.

This paper addresses the problem of insufficient data to determine the covariance function based on estimates of the first and second order of random fields - i.e. the mean value and the empirical semivariogram - that describe the spatial variability.
Methods like the least-squares (LSQ) method require a large dataset in order to accurately determine these characteristics of the covariance function by fitting a variogram model on the empirical data.

Bayesian updating techniques enable to estimate the parameters of the covariance function more rigorously and with less ambiguity as these can be used to update previously obtained information regarding parameters of similar random fields. Markov chain Monte Carlo (MCMC) simulations can be used to incorporate Bayesian updating based on limited samples in the parameter estimation.

As a common simplification the investigated random fields are assumed to be Gaussian random fields with homogeneous, isotropic and ergodic properties. Gaussian fields are important for two main reasons. First, the Gaussian distribution is well-studied, which makes the calculations more feasible and second the central limit theorem states that the net result of many small-order effects is approximately Gaussian (Cressie, 1993). Gaussian random fields are completely defined by their first and second order characteristics.

The proposed procedure is evaluated through numerical simulations of Gaussian random fields with known characteristics. Samples are taken and 
are used to compute empirical semi-variograms. A semi-variogram model is than fitted on the empirical semi-variogram while considering prior information about the model parameters.

Since it is typically difficult or even impossible to analytically solve the expression for the posterior distribution, MCMC simulations are used. In the following first some basic concept are explained, an adapted Metropolis-Hastings algorithm is established in order to conduct the MCMC simulations. Finally the suggested Bayesian updating method is compared with the LSQ method.

\section{RANDOM FIELDS}

\subsection{Basic concepts}

A random field $\{X(\boldsymbol{t}), \boldsymbol{t} \in \Omega\}$ is a function whose values are random variables for any position $t$ in the domain $\Omega \in \mathbb{R}^{\mathrm{d}}$. A representation of a random field is denoted by a deterministic function $x(t)$. Random fields can be decomposed in a trend surface $m(\boldsymbol{t})$ and a residual variation with mean zero for each position $\boldsymbol{t}$. This residual variation usually exhibits a spatial correlation, which is represented by a covariance function $B_{k l}\left(\boldsymbol{t}_{k}, \boldsymbol{t}_{l}\right)$. In general this covariance function can be different for every $\boldsymbol{t}_{k}, \boldsymbol{t}_{l}$. Random fields whose values follow a normal distribution are called Gaussian random fields. Gaussian random fields are second order stationary, thus they can be specified by a constant mean $\mu$ and a deterministic covariance function $B\left(\boldsymbol{t}_{k}, \boldsymbol{t}_{l}\right)$. Because homogeneous, isotropic and ergodic properties were assumed, the covariance function $B(\tau)$ is only dependent on the distance $\tau$ in the random field. Note that $B(0)$ is the variance $\sigma^{2}$ of the field, since it defines the covariance of a variable with itself.

There are different models available for the covariance function. Commonly used models are the exponential, squared exponential and the Matérn covariance function. Both the exponential and squared exponential require only one model parameter that defines a measure for the distance over which the random field is correlated. The Matérn covariance model incorporates an additional parameter $v$, which is called the smoothness parameter.

In this work the exponential model will be used:

$B(\tau)=\sigma^{2} \exp \left(-\frac{|\tau|}{\theta}\right)$

where $\sigma^{2}$ is the variance of the field, $\tau$ is the distance in the field and $\theta$ is a scale-parameter that defines the shape of the exponential covariance model.

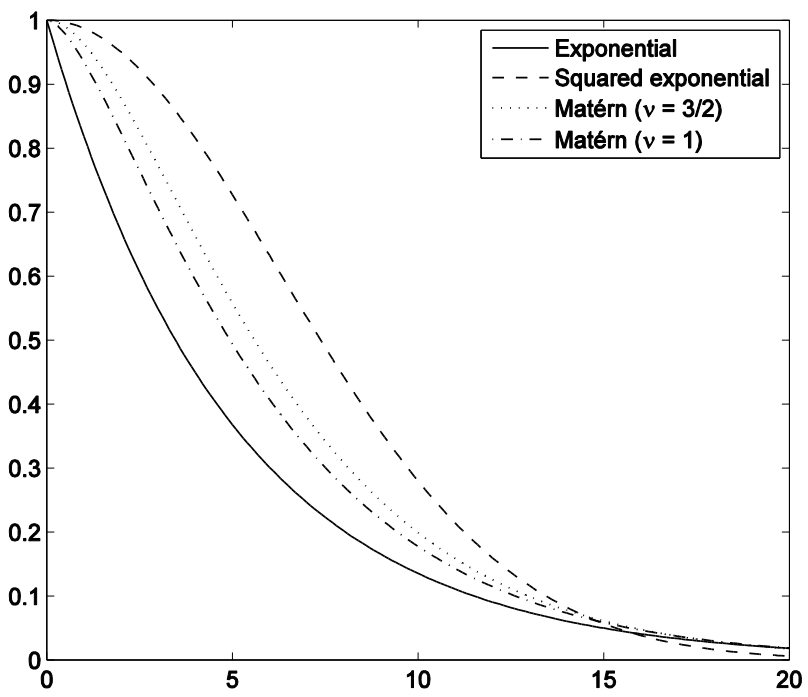

Figure 1. Different covariance functions for $\beta=5$ and $\sigma^{2}=1$

The correlation length $\beta$ is introduced. It is a measure of the distance over which the random field is correlated. It is defined as the centroid of the surface under $B(\tau)$ (Baecher and Christian, 2003):

$\beta=\frac{\int \tau B(\tau) \mathrm{d} \tau}{\int B(\tau) \mathrm{d} \tau}$

For the exponential model the correlation length $\beta$ is equal to the scale parameter $\theta$.

In spatial data analysis it is more common to use the variogram $2 \gamma(\tau)$ or the semi-variogram $\gamma(\tau)$ instead of the covariance function. The variogram like the covariance function - is a function describing the degree of spatial variation. The variogram, rather than the covariance function, is used in spatial data analysis because the empirical variogram does not rely on the knowledge of the mean $\mu$ of the random field. Because Gaussian fields are second order stationary the following relation between the covariance function and the variogram holds:

$2 \gamma(\tau)=2(B(0)-B(\tau))$

\section{BAYESIAN ESTIMATION OF RESPONSE SURFACE PARAMETERS}

Bayesian estimation of linear model parameters is well-described in literature, e.g. in (Box and Tiao, 1992, Gamerman and Lopes, 2006, Gelman et al., 2003, Ghosh et al., 2010, Gregory, 2005, Lee, 2012). However, in most cases observational data is 
modelled as a nonlinear combination of multiple model parameters and input variables. Available literature on Bayesian estimation of nonlinear model parameters is rather limited (Gelman et al., 2003, Gregory, 2005).

Assume that the true value of the response variable $\tilde{y}$ can be predicted by a mathematical model $M$, which is a nonlinear function of $R$ parameters $\beta_{r}(r=1, \ldots, R)$ and depends on a vector $\boldsymbol{x}$, which represents a $m$-dimensional set of input variables. If this model would be perfect and the true values $\widetilde{\boldsymbol{x}}$ are exactly known, the model would be able to predict the true response value $\tilde{y}$ exactly. However, due to the existence of model and measurement uncertainties, the true value is given by:

$\tilde{y}=M(x)+\varepsilon$

where the error term $\varepsilon$ can be considered as a Gaussian distributed variable with mean 0 and with an unknown variance $\sigma_{\varepsilon}^{2}$, representing the uncertainties. The variance $\sigma_{\varepsilon}^{2}$ of the error term is assumed to be constant over the domain of the input variables.

$\varepsilon \propto N\left(0, \sigma_{\varepsilon}^{2}\right)$

If $N$ independent response variables $y_{i}$ are available for $N$ sets of corresponding input vectors $\boldsymbol{x}_{i}$, then the likelihood of the experimental data can be written as:

$L\left(y_{1}, \ldots, y_{N} \mid \sigma_{\varepsilon}, \boldsymbol{\beta}\right)=\prod_{i=1}^{N} \frac{1}{\sigma_{\varepsilon}} \phi\left(\frac{y_{i}-M\left(x_{i}\right)}{\sigma_{\varepsilon}}\right)$

where $\phi(\cdot)$ is the probability density function (PDF) of the standard normal distribution.

The prior information is given as the joint prior distribution $f_{B}^{\prime}\left(\sigma_{\varepsilon}, \boldsymbol{\beta}\right)$ of the standard deviation $\sigma_{\varepsilon}$ of the error term and the model parameters $\boldsymbol{\beta}$. This prior distribution can be updated towards a posterior distribution $f_{B}^{\prime \prime}\left(\sigma_{\varepsilon}, \boldsymbol{\beta}\right)$ by using Bayes' theorem:

$$
f_{B}^{\prime \prime}\left(\sigma_{\varepsilon}, \boldsymbol{\beta}\right)=c f_{B}^{\prime}\left(\sigma_{\varepsilon}, \boldsymbol{\beta}\right) L\left(y_{1}, \ldots, y_{N} \mid \sigma_{\varepsilon}, \boldsymbol{\beta}\right)
$$

with $C$ a normalizing constant and $B$ the domain of the parameters $\left(\sigma_{\varepsilon}, \boldsymbol{\beta}\right)$ that have to be updated. Equation 7 can be difficult or impossible to solve analytically. Therefore MCMC simulations are applied (i.e. using the Metropolis-Hastings algorithm (Gelman et al., 2003)) to estimate values for the model parameters $\boldsymbol{\beta}$ and the standard deviation $\sigma_{\varepsilon}$ of the error term.

\section{ADAPTED METROPOLIS-HASTINGS ALGORITHM}

Markov chain Monte Carlo methods (MCMC) allow obtaining samples from probability distributions based on the construction of a Markov chain. A Markov chain is defined as a sequence of random variables $x_{i}$ for which the distribution of $x_{i}$ depends only on the previous sample $x_{i-1}$, i.e. not on $x_{i-2}, x_{i-3}$, etc. (Gelman et al., 2003). These methods allow to draw a discrete-time homogeneous chain of samples from the posterior distribution (Perrin et al., 2007). The idea is to generate iteratively samples of a Markov chain, which asymptotically behaves as the probability density function (PDF) that has to be sampled. More specifically, the Metropolis-Hastings algorithm (Hastings, 1970, Nicholas et al., 1953) is commonly used for generating such Markov chains.

Considering a certain PDF $f_{X}(\boldsymbol{x})$ that is a function of an input vector $\boldsymbol{x}, \mathrm{MCMC}$ realizations $\boldsymbol{x}_{s}$ are generated sequentially and independently, starting from an arbitrary chosen starting vector $\boldsymbol{x}_{0}$. In each step, the transition between the states $\boldsymbol{x}_{s}$ and $\boldsymbol{x}_{s+1}$ is given according to (see e.g. (Gelman et al., 2003)):

$$
\boldsymbol{x}_{s+1}=\left\{\begin{array}{ccc}
\tilde{\boldsymbol{x}} \propto & q\left(\tilde{\boldsymbol{x}} \mid \boldsymbol{x}_{s}\right) & \alpha\left(\boldsymbol{x}_{s}, \tilde{\boldsymbol{x}}\right) \\
\boldsymbol{x}_{s} & \text { else }
\end{array}\right.
$$

where $\widetilde{\boldsymbol{x}}$ is a candidate vector, $q\left(\widetilde{\boldsymbol{x}} \mid \boldsymbol{x}_{s}\right)$ is called the transition or jumping distribution and the acceptance probability $\alpha\left(\boldsymbol{x}_{s}, \widetilde{\boldsymbol{x}}\right)$ is given by (see e.g. (Gelman et al., 2003)):

$\alpha\left(\boldsymbol{x}_{s}, \widetilde{\boldsymbol{x}}\right)=\min \left\{1, \frac{f_{X}(\widetilde{\boldsymbol{x}})}{f_{X}\left(\boldsymbol{x}_{S}\right)} \frac{q\left(\boldsymbol{x}_{s} \mid \widetilde{\boldsymbol{x}}\right)}{q\left(\widetilde{\boldsymbol{x}} \mid \boldsymbol{x}_{S}\right)}\right\}$

Practically, in order to select a candidate $\tilde{\boldsymbol{x}}$, a random number $r_{s}$ is generated from a uniform distribution $U[0 ; 1]$. The candidate $\widetilde{x}$ is accepted as the next realization of $f_{X}(\boldsymbol{x})$ with a probability $\alpha\left(\boldsymbol{x}_{s}, \widetilde{\boldsymbol{x}}\right)$ in case $r_{s} \leq \alpha\left(\boldsymbol{x}_{s}, \widetilde{\boldsymbol{x}}\right)$ or rejected in the other case. In this manner, a sequence of random draws $\boldsymbol{x}_{s}$ from $f_{X}(\boldsymbol{x})$ is generated, even when no analytical solution is available for $f_{X}(\boldsymbol{x})$.

In case of the Bayesian estimation of response surface parameters, the transition between two estimates $\left(\sigma_{\varepsilon, s}, \boldsymbol{\beta}_{s}\right)$ and $\left(\sigma_{\varepsilon, s+1}, \boldsymbol{\beta}_{s+1}\right)$ for the posterior set of response surface parameters can be rewritten as:

$$
\begin{aligned}
& \left(\sigma_{\varepsilon, s+1}, \boldsymbol{\beta}_{s+1}\right)= \\
& \left\{\begin{array}{cc}
\left(\tilde{\sigma}_{\varepsilon}, \widetilde{\boldsymbol{\beta}}\right) \propto q\left(\tilde{\sigma}_{\varepsilon}, \widetilde{\boldsymbol{\beta}} \mid \sigma_{\varepsilon, s}, \boldsymbol{\beta}_{s}\right) & \psi \\
\left(\sigma_{\varepsilon, s}, \boldsymbol{\beta}_{s}\right) & \text { else }
\end{array}\right.
\end{aligned}
$$


where $q\left(\tilde{\sigma}_{\varepsilon}, \widetilde{\boldsymbol{\beta}} \mid \sigma_{\varepsilon, s}, \boldsymbol{\beta}_{s}\right)$ is the transition distribution and $\psi$ the joint acceptance probability. A common choice for this transition distribution is the random walk algorithm, more specifically by adding a random increment $\boldsymbol{s}=\left(\varsigma_{0}, \varsigma_{1}, \ldots, \varsigma_{R}\right)$ to the previous estimate according to:

$$
\left(\tilde{\sigma}_{\varepsilon}, \widetilde{\boldsymbol{\beta}}\right)=\left(\sigma_{\varepsilon, s}, \boldsymbol{\beta}_{s}\right)+\boldsymbol{\varsigma}
$$

with $\boldsymbol{\varsigma}$ a random vector that does not depend on the previous chain. In practice, it is common to choose the values $S_{i}$ according to a normal or uniform distribution with mean 0 and variance $\sigma_{\varsigma}^{2}$. The random walk algorithm results in a symmetrical transition $q\left(\sigma_{\varepsilon, s}, \boldsymbol{\beta}_{s} \mid \tilde{\sigma}_{\varepsilon}, \widetilde{\boldsymbol{\beta}}\right)=q\left(\tilde{\sigma}_{\varepsilon}, \widetilde{\boldsymbol{\beta}} \mid \sigma_{\varepsilon, s}, \boldsymbol{\beta}_{s}\right)$. Hence, the transition distribution does not appear in the expression for the joint acceptance probability:

$\psi\left(\sigma_{\varepsilon, s}, \boldsymbol{\beta}_{s} \mid \widetilde{\sigma}_{\varepsilon}, \widetilde{\boldsymbol{\beta}}\right)=\min \left\{1, \frac{f_{B}^{\prime \prime}\left(\widetilde{\sigma}_{\varepsilon}, \widetilde{\boldsymbol{\beta}}\right)}{f_{B}^{\prime \prime}\left(\sigma_{\varepsilon, s}, \boldsymbol{\beta}_{s}\right)}\right\}$

Further, the probability $\psi$ is the joint acceptance probability based on the prior probability and the likelihood function or in other words the probability that a random sample $u_{P} \propto U[0 ; 1]$ from a uniform distribution is accepted according to the prior distribution and that a random sample $u_{L} \propto U[0 ; 1]$ is accepted according to the likelihood function. This probability is generalized for Bayesian estimation of response surface parameters according the following equations:

$\psi=\operatorname{Prob}\left[\left(u_{P}<\alpha_{P}\right) \cap\left(u_{L}<\alpha_{L}\right)\right]$

$\alpha_{P}=\min \left\{1, \frac{f_{B}^{\prime}\left(\widetilde{\sigma}_{\varepsilon}, \widetilde{\boldsymbol{\beta}}\right)}{f_{B}^{\prime}\left(\sigma_{\varepsilon, s}, \boldsymbol{\beta}_{s}\right)}\right\}$

$\alpha_{L}=\min \left\{1, \frac{L\left(y_{1}, \ldots, y_{N} \mid \widetilde{\sigma}_{\varepsilon}, \widetilde{\boldsymbol{\beta}}\right)}{L\left(y_{1}, \ldots, y_{N} \mid \sigma_{\varepsilon, s}, \boldsymbol{\beta}_{S}\right)}\right\}$

with the likelihood $L\left(y_{1}, \ldots, y_{N} \mid \ldots\right)$ according to Equation 6 in case of independent response values.

The convergence speed of the Markov chain is strongly influenced by the random increment $\boldsymbol{\varsigma}$ (each $S_{i}$ either uniform or standard normally distributed) and more specifically by its standard deviation $\sigma_{\varsigma}$. This standard deviation has to make sure that the domain over which $f_{B}^{\prime \prime}$ is sampled is properly explored. If the standard deviation $\sigma_{\varsigma}$ is small, then the Markov chain will converge slowly, because more iterations are needed to explore the entire domain of $f_{B}^{\prime \prime}$. However, if $\sigma_{\varsigma}$ is too large, then the chain will also converge slowly, because the acceptance probability is too low. Thus, it is important to make sure that the Markov chain has enough iterations to represent the function $f_{B}^{\prime \prime}$ accurately and to detect the length of the burn-in period (the period in the Markov chain where stationarity is not yet obtained) so that these initial values can be deleted from the samples for $f_{B}^{\prime \prime}$.

\section{BAYESIAN ESTIMATION OF THE CORRELATION LENGTH}

The method described in sections 3 and 4 can be used to estimate the covariance function of a random field based on limited measurements by fitting a semi-variogram model to the empirical semivariogram. In case of an exponential of squared exponential covariance function the only unknown parameter is the scale parameter $\theta$ which is equal to the correlation length $\beta$. The exponential covariance function is a nonlinear model with one model parameter and one input variable.

Rewriting Equation 4 in terms of the semivariogram yields:

$\bar{\gamma}(\tau)=\gamma(\tau \mid \beta)+\varepsilon$

where $\bar{\gamma}(\tau)$ is the empirical semi-variogram, $\gamma(\tau \mid \beta)$ the semi-variogram model and $\varepsilon$ the error term as defined in Equation 5.

There are different methods available to compute an empirical semi-variogram based on measurement results (Cressie, 1993). In this paper the method-ofmoments estimator defined by Matheron (1962) is adopted:

$\bar{\gamma}(\tau)=\frac{1}{|T(\tau)|} \sum_{T(\tau)}\left(x\left(\boldsymbol{t}_{i}\right)-x\left(\boldsymbol{t}_{j}\right)\right)^{2}$

$T(\tau)=\left\{\left(x\left(\boldsymbol{t}_{i}\right), x\left(\boldsymbol{t}_{j}\right)\right) ;\left\|x\left(\boldsymbol{t}_{i}\right)-x\left(\boldsymbol{t}_{j}\right)\right\|=\tau\right\}$

where $|T(\tau)|$ is the number of elements in the set $T(\tau)$.

$\bar{\gamma}(\tau)$ is a discrete function as there are only a finite number of distances $\tau$ between the different measurement points $x\left(\boldsymbol{t}_{i}\right), x\left(\boldsymbol{t}_{j}\right)$. In order to obtain more measurement points at a same distance $\tau$ apart per set $T(\tau)$, similar distances are grouped in distance classes by allowing a tolerance $\epsilon$ on the distance $\tau$. Hence, two measurements are element of $T(\tau)$ if:

$\left|\left\|x\left(\boldsymbol{t}_{i}\right)-x\left(\boldsymbol{t}_{j}\right)\right\|-\tau\right|<\epsilon$ 
The tolerance $\epsilon$ should be chosen in a manner that there are enough elements in each set $T(\tau)$ but not at the expense of the overall representation of the semivariogram (e.g. if the tolerance $\epsilon$ is chosen so that every combination complies with Equation 19 than the semi-variogram will be constant, which is most definitely not an accurate result). It is pointed out that due to the nature of the method-of-moments estimator the semi-variogram can show considerable variation for different realisations of a random field. Of course less measurement points lead to more variation of the semi-variogram.

The likelihood function introduced in Equation 6, rewritten in terms of the semi-variogram yields:

$$
L=\prod_{i=1}^{M} \frac{1}{\sqrt{2 \pi} \sigma_{\varepsilon}} \exp \left(-\frac{1}{2} \frac{\left(\bar{\gamma}\left(\tau_{i}\right)-\gamma\left(\tau_{i} \mid \beta\right)\right)^{2}}{\sigma_{\varepsilon}^{2}}\right)
$$

with $M$ the number of defined distances $\tau$ between the different measurement points $x\left(\boldsymbol{t}_{i}\right), x\left(\boldsymbol{t}_{j}\right)$. The acceptance probabilities $\alpha_{P}$ and $\alpha_{L}$ (see Equations 14 and 15) are given by:

$\alpha_{P}=\min \left\{1, \frac{f_{B}^{\prime}\left(\widetilde{\sigma}_{\varepsilon}, \widetilde{\beta}\right)}{f_{B}^{\prime}\left(\sigma_{\varepsilon, s}, \beta_{s}\right)}\right\}$

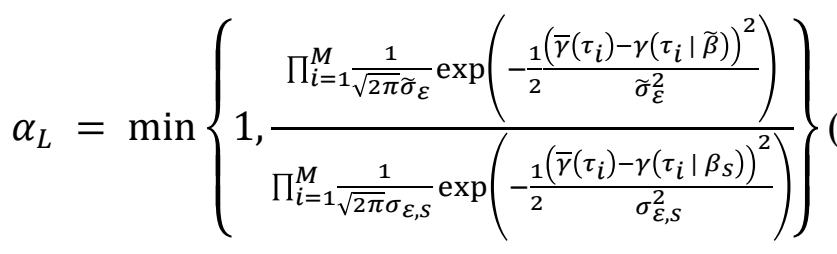

with $f_{B}^{\prime}\left(\sigma_{\varepsilon}, \beta\right)$ the joint prior distribution for the correlation length and the standard deviation of the error term.

The adapted Metropolis-Hastings algorithm can now be used to sample the posterior distribution for the correlation length $\beta$. The estimation for the correlation length is hence given by the expectance of the posterior distribution. Further, this method will be referred to as the MCMC method

\section{POSITION OF THE MEASUREMENT POINTS}

In order to verify if the position of the measurement points influences the result of the MCMC method two different sample pattern are considered. A pattern with a constant distance between the measurement points and a pattern where the distance between the measurement points increases exponentially. A conceptual representation of these sample pattern as is shown in Figure 2. For the regular pattern a larger amount of measurements for each distance $\tau_{i}$ is available, while in the case of the logarithmic regular pattern a larger amount of different distances $\tau_{i}$ is available. For each pattern 25 samples were taken over the same span from 50 different realizations of a standard normal distributed random field with an exponential covariance function with correlation length $\beta=10$. A lognormal prior distribution, with $\mu_{\beta}=10$ and $\sigma_{\beta}=1$, for the correlation length and a uniform distribution for the standard deviation of the error term were considered. Results are shown in Figure 3.
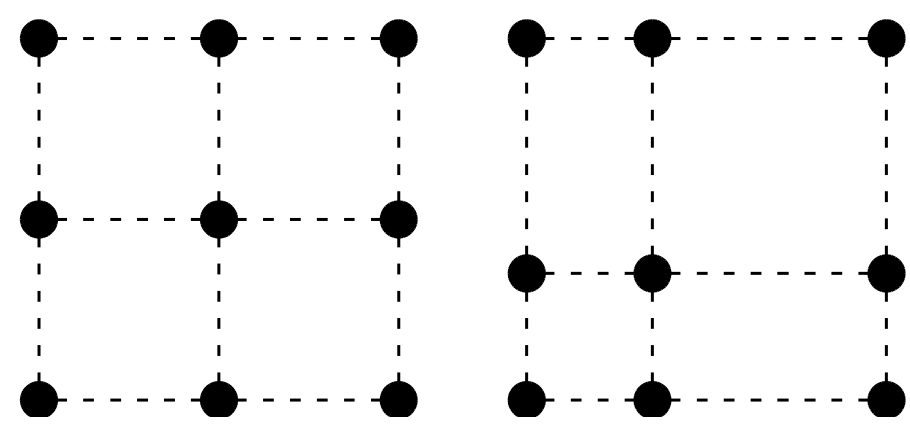

Figure 2. Examples of possible sample patterns to be applied in the random field. Left a regular pattern and right a logarithmic regular pattern.

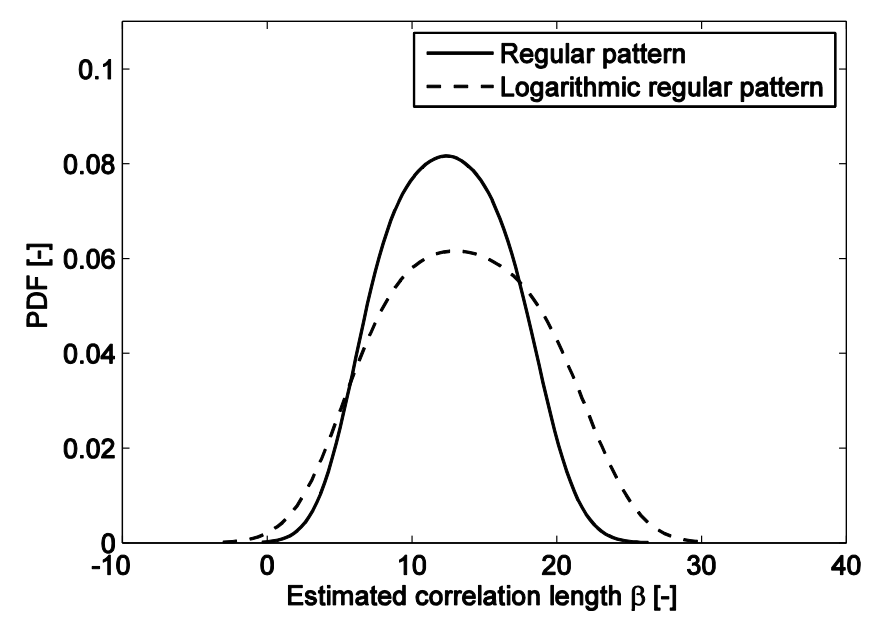

Figure 3 Distributions of the estimated correlation length for the regular sample pattern and the logarithmic sample pattern.

The regular sample pattern consequently results in a slightly smaller confidence interval for the estimated correlation length, because more measurements are available for a few distances $\tau_{i}$. However, the difference between the two sample patterns is small. This small difference can be attributed to the fact that the empirical semi-variogram shows considerable variation if only a few measurement points are available. Hence, the influence of the position of the samples is far less important than the influence of the uncertain nature of the semivariogram. 


\section{COMPARISON BETWEEN THE LSQ AND THE MCMC METHOD}

In order to compare the LSQ method and the proposed MCMC method, both methods are applied to find the correlation length of a random field on a grid with 32 by 32 positions. In the case of the MCMC method 25 measurement points are considered, while for the LSQ method either 25 (8 positions between each sample point) or 1024 (all positions of the random field) measurements are considered. Samples were taken from 100 different realizations of a standard normal distributed random field with an exponential covariance function with correlation length $\beta=10$ according to a regular sample pattern. The LSQ method solves following equation to estimate the correlation length:

$\beta=\arg \min \sum_{i=1}^{M}\left(\gamma\left(\tau_{i}\right)-\bar{\gamma}\left(\tau_{i} \mid \beta\right)\right)^{2}$

where $\gamma(\cdot)$ is the empirical semi-variogram and $\bar{\gamma}(\cdot \mid \beta)$ is de semi-variogram model.

In case of the MCMC method a lognormal prior distribution, with $\mu_{\beta}=9$ and $\sigma_{\beta}=1$, for the correlation length and a uniform distribution for the standard deviation of the error term were considered. Notice that the prior distribution for the correlation length is biased. Results are shown in Figure 4.

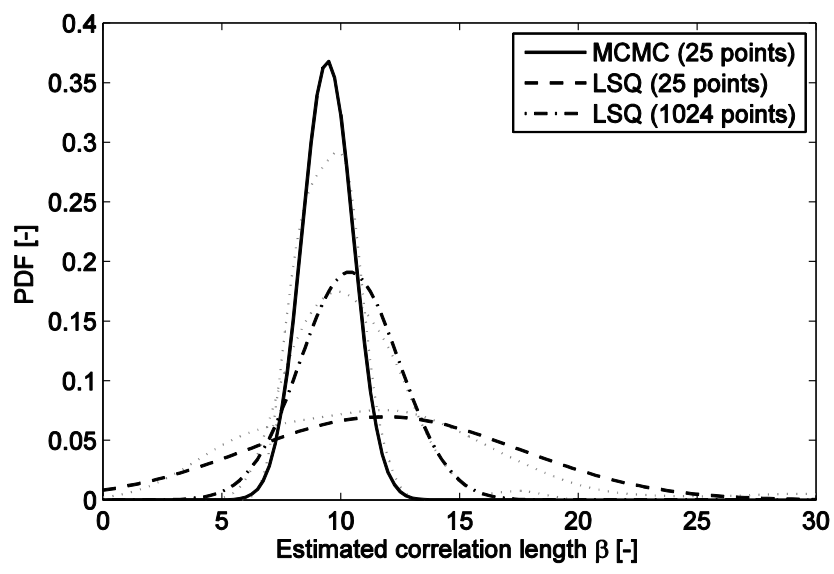

Figure 4. Distributions of the estimated correlation length using the MCMC and LSQ method. (The kernel density estimations are represented by the dotted lines.)

Compared to the LSQ method, the estimated correlation length using the MCMC method shows less variation even when compared to the LSQ method that makes use of a large number of measurements. As such large datasets are often impossible to achieve for certain material properties, this moreover underlines the importance of using prior knowledge.

\section{CONCLUSIONS}

A common problem when estimating the correlation length in common engineering problems is a lack of available data which makes traditional methods to estimate the spatial correlation such as the least squares method difficult to apply.

A Bayesian response surface method based on Markov Chain Monte Carlo (MCMC) simulations is developed in order to estimate the correlation length (i.e. covariance function) of random fields from empirical semi-variograms using Bayesian updating of prior (vague of informative) information. This prior information should be based on previous test results or expert judgment. Available measurement data, even limited is used to update these prior assumptions.

This MCMC method is found out to be less susceptible to the increased uncertainty resulting from the lack of data. This can be mainly attributed due to the incorporation of prior information. Simulations have shown that the proposed response surface method gives similar results compared to least-squares methods if sufficient data is available.

Large datasets are often impossible to achieve for certain material properties in structural engineering applications, hence indicating the importance of the proposed Bayesian response surface updating model.

\section{REFERENCES}

Baecher, G. B. \& Christian, J. T. (2003) Reliability and Statistics in Geotechnical Engineering, John Wiley \& Sons.

Box, G. E. P. \& Tiao, G. C. (1992) Bayesian inference in statistical analysis, Wiley.

Cressie, N. a. C. (1993) Statistics for spatial data, J. Wiley.

Gamerman, D. \& Lopes, H. F. (2006) Markov Chain Monte Carlo: Stochastic Simulation for Bayesian Inference, Second Edition, Taylor \& Francis.

Gelman, A., Carlin, J. B., Stern, H. S. \& Rubin, D. B. (2003) Bayesian Data Analysis, Chapman \& Hall/CRC.

Ghosh, J. K., Delampady, M. \& Samanta, T. (2010) An Introduction to Bayesian Analysis: Theory and Methods, Springer.

Gregory, P. (2005) Bayesian Logical Data Analysis for the Physical Sciences: A Comparative Approach with Mathematica ${ }^{\circledR}$ Support, Cambridge University Press.

Hastings, W. K. (1970) Monte Carlo sampling methods using Markov chains and their applications. Biometrika, 57, 97-109.

Lee, P. M. (2012) Bayesian Statistics: An Introduction, Wiley.

Matheron, G. (1962) Traité de géostatistique appliquée, Éditions Technip.

Nicholas, M., Arianna, W. R., Marshall, N. R., Augusta, H. T. \& Edward, T. (1953) Equation of State Calculations by Fast Computing Machines. The Journal of Chemical Physics, 21, 1087-1092.

Perrin, F. S., Pendola, M. \& De Rocquigny, E. (2007) Comparison of Markov chain Monte Carlo simulation 
and FORM-based approach for Bayesian updating of mechanical models. 10th int. Conf. on Applications of Statistics and Probaility in Civil Engineering. Tokyo, Taylor \& Francis Group. 